FACULDADE DE CIÊ NCIAS ECONÔ MICAS DA UFRGS

REESTRUTURAÇÁ O DA PRODUÇÁ O AGRICOLA NO RIO GRANDE DO SUL, DE 1970 A 1996 E UMA ESTIMAÇȦ O PARA 2012 TIAGO WICKSTROM ALVES

A CRIMINALIDADE NA RECIAO METROPOLTANA DE SALVADOR JOSÉ CARRERA FERNANDEZ, LUIZ FERNANDO LOBO

COMPEIITIVIDADE INTERNACIONAL EM SOFTWARE: UM ESTUDO SOBRE A EXPERIÊNCIA DE FLORIANÓ POLIS HOVÉDO NUNES LINS

MODELOS DE ORGANIZAÇẢ O E REPARTIÇẢ O DE RENDAS NA CADEIA PRODUTIVA DO GÁS NATURAL.

HELder QUEIROZ PINTO Jú NIOR, RODOLFO TORRES dOS SANTOS

REGIMES MONETÁ RIOS E DIVIDA PÚ BLICA: UMA ANÁ LISE DE MECANISMOS ALTERNATIVOS DE COORDENAÇÃO MACROECONÓ MICA

manoel Carlos de Castro Pires

ECONOMIA DOS RECURSOS NATURAIS E SEUS INDICADORES DE ESCASSEZ: UMA QUESTÃ O DE SUSTENTABILIDADE ANDREA SALES SOARES DE AZEVEDO MELO

O ECOPROTECIONISMO AGRICOLA NA UNIĀ O EUROPEIA E SEUS POSSIVEIS IMPACTOS SOBRE A ECONOMIA BRASILEIRA Rodrigo daniel Feix, Clá udio R. Fóffano Vasconcelos

PADRÓ ES DE DESENVOLVIMENTO, FUNÇ Ó ES ESTATAIS E ENDIVIDAMENTO NO CAPITALISMO CONTEMPORÁNEO paulo Balanco, Eduardo Costa Pinto

A INTERNACIONALIZAÇÃO DO VAREJO A PARTIR DOS CASOS WAL-MART E CARREFOUR

Armando joảo dalla Costa

UMA ANÁ LISE ECONOMÉTRICA DO FUTEBOL BRASILEIRO ARI Francisco de Araujo JR, Clá udio D. SHIKIDA, LEONARDO M. MONASTERIO

O BRASIL E A ECONOMIA INTERNACIONAL: RECUPERAÇÃ O E DEFESA DA AUTONOMIA NACIONAL

RESENHA DE ALEXANDRE CÉ SAR CUNHA LEITE

REGIMES MONETÁ RIOS: TEORIA E A EXPERIÊNCIA DO REAL ResenHa de Fernando Ferrari Filho

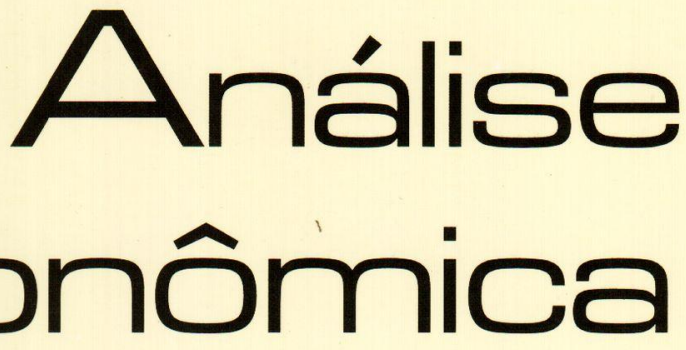


Universidade Federai. do Rio Grande do Sul Reitor: Prof José Carlos Ferraz Hemnemann

Facul dade de Cuéncias Economicas

Diretor: Prof. Gentil Corazza

Ceniro de Estudos f Prequisas Economicas

Diretor: Prof. Lovois de Andrade Miguel

Depariamenio de Crências Economicas

Chefe: Prof Ricardo Dathein

Departamento de Cîncias Coniábeis f A tuaria Chefe: Prof. Ceno Odilo Kops

Curso de Pós-Graduaçó em Economia

Coordenador: Prof. Fernando Ferrari Filho

Curso de Pos-Grainaça em Desenvolvimento RuRAI

Coordenador: Prof. Paulo Dabdab Waquil

Conseluo Eni tokial: André Moreira Cunha (UFRGS) Carlos G. A. Mielitz Netto (UFRGS), Carlos Henrique Hom (UFRGS). Eduardo A. Maldonado Filho (UFRGS). Eleutério F S. Prado (USP), Eugênio Lagemann (UFRGS), Fernando Cardim de Carvalho (UFRJ). Femando Ferrari Filho (UFRGS), Fernando de Holanda Barbosa (FGV/RJ), Flávio Augusto Ziegelmann (UFRGS), Flávio Vasconcellos Comin (UFRGS), Gentil Corazza (UFRGS), Giácomo Balbinot to Neto (UFRGS), Gustavo Franco (PUC/RJ) Hélio Henkin (UFRGS), Jan A. Kregel (UNCTAD), João Rogério Sanson (UFSC), Joaquim Pinto de Andrade (UnB), Júlio César Oliveira (UFRGS), Luiz Estrella Faria (UFRGS), Luiz Paulo Ferreira Nogueról (UFRGS), Marcelo S Portugal (UFRGS), Maria Alice Lahorgue (UFRGS), Octávio Augusto Camargo Conceicão (UFRGS), Paul Davidson (University of Tennessee), Paulo D. Waquil (UFRGS), Pedro C. D. Fonseca (UFRGS), Philip Arestis (University of Cambridge), Ricardo Dathein (UFRGS) Ronald Otto Hillbrecht (UFRGS), Sabino da Silva Porto Jr (UFRGS), Sérgio M. M. Monteiro (UFRGS), Stefano Florissi (UFRGS) e Werner Baer (University of IIIinois at Urbana - Champaign)

Comissão Emroniai: Eduardo Augusto Maldonado Filho, Fernando Ferrari Filho, Hélio Henkin. Marcelo Savino Portugal, Paulo Dabdab Waquil e Sérgio Marley Modesto Monteiro.

EnIrok: Sérgio Marley Modesto Monteiro

Ediror Adjun ro: Hélio Henkin

Secretário: Emerson Douglas Neves

REVISÃo DE IEX ros: Vanete Ricacheski

Emioração: Núcleo de Editoração e Criação da

Gráfica da UFRGS - Janaína Horn e Junia Saedt

Fundador: Prof Antonio Carlos Santos Rosa
Os materiais publicados na revista Análise Econômica são da exclusiva responsabilidade dos autores. É permitida a reprodução total ou parcial dos trabalhos, desde que seja citada a fonte. Aceita-se permuta com revistas congêneres. Aceitam-se, também, livros para divulgacão, elaboraça de resenhas e recensões Toda correspondência: material para publicação (vide normas na terceira capa), assinaturas e permutas devem ser dirigidos ao seguinte destinatário:

Prof. Sérgio Marley Modesto Monteiro Revista Andilse Económica - Av. João Pessoa, 52 CEP 90040-000 PORTO ALEGRE - RS, BRASIL Telefones: (051) $33163513 / 33164164$

Fax: (051) 33163990 - E-mail: rae@vortex ufrgs.br

A Revista Análise Econômica agradece a colaboração dos pareceristas do número 43, abaixo relacionados:

Adalmir Antonio Marquetti

André Moreira Cunha

Carlos José Caetano Bacha

Carlos Mielitz. Netto

Clailton Ataídes de Freitas

Claudio Roberto Fóffano Vasconcelos

Eduardo Ernesto Filippi

Eduardo Pontual Ribeiro

Eleuterio Fernando da Silva Prado

Fernando J Cardim de Carvalho

Fernando Augusto Mansor de Mattos

Fernando Ferrari Filho

Flávio Tosi Feijó

Flavio Vilela Vieira

Francisco Paulo Cipolla

Gentil Corazza

Gíacomo Balbinotto Neto

Helio Henkin

Jefferson Andronio Ramundo Staduto

João de Deus Sicsú Siqueira

José Carrera Fernandes

Leonardo Monteiro Monasterio

Luís Fernando de Paula

Luis Roberto Nascimento

Manoel Carlos de Castro Pires

Octavio Augusto Camargo Conceição

Patrízia Raggi Abdallah

Robson Antonio Grassi

Ronald Otto Hilbrech

Tiago Wickstrom Alves

Tito Belchior Silva Moreira

Análise Econônica

Ano 23, n ${ }^{\circ} 44$, setembro, 2005 - Porto Alegre

Faculdade de Ciências Economicas, UFRGS, 2000

Periodicidade semestral, março e setembro

1. Teoria Econômica - Desenvolvimento Regional -

Economia Agrícola - Pesquisa Teórica e Aplicada -

Periódicos. I.. Brasil

Faculdade de Ciências Econômicas,

Universidade Federal do Rio Grande do SuI

CDD 330.05

CDU $33(81)(05)$ 


\title{
Modelos de organização e repartição de rendas na cadeia produtiva do gás natural
}

\author{
Helder Queiroz Pinto Júnior * \\ Rodolfo Torres dos Santos*
}

\begin{abstract}
Resumo: Neste artigo apresentam-se critérios para comparação de modelos de organização vertical de cadeias produtivas a partir da experiência da indústria de gás natural. No modelo I, forma tradicional de organização da indústria, o encadeamento das transações comerciais coincide com o da produção. $O$ modelo II difere-se do modelo I pela presença do segmento de carregadores independentes. Os critérios utilizados para comparar estas duas alternativas são: i) o impacto sobre o preço ao consumidor final; ii) o equilíbrio na repartição da renda líquida entre os segmentos da cadeia de valor. O principal resultado é que, atendendo ao critério de repartição equânime das rendas, o modelo II garante também garante a eficácia em relação ao I em termos de menor preço ao consumidor final.
\end{abstract}

\begin{abstract}
This paper compares two models of value chain organization based on the natural gas industry case. In the model I, the traditional production chain organization, commercial relationships are made in the same way of the production chain. The model II is different because of the presence of independent carriers. The comparison between those models considers (i) the outcome in the consumers price and (ii) the equilibrium of the rent distribution among value chain segments. The main result of this paper is that if the rent repartition is equal among value chain segments, then the model II promotes lower consumers price than the model I.
\end{abstract}

Keywords: mark-up, estrutura de mercado, integração vertical, gás natural.

Código JEL: L10, L50, L90.

\section{Introdução}

Este artigo objetiva apresentar critérios para comparação de modelos de organização vertical de cadeias produtivas a partir da experiência da indústria de gás natural e as possíveis conseqüências para a repartição da renda entre os diferentes agentes econômicos. Os dois modelos de organização aqui contemplados representam a diversidade de formas organizacionais das transações nessa 
indústria. No modelo I, forma tradicional de organização da indústria, fortemente marcado pela integração vertical das atividades, o encadeamento das transações comerciais coincide com o da produção. O modelo II difere-se do precedente pela presença do segmento de carregadores independentes; estes agentes são responsáveis pela intermediação comercial entre a produção, o transporte e a distribuição.

Os critérios utilizados para comparar estas duas alternativas são: i) o impacto sobre o preço ao consumidor final; ii) o equilíbrio na repartição da renda líquida entre os segmentos da cadeia de valor. O principal resultado é que, atendendo ao critério de repartição equânime das rendas, o modelo Il garante também garante a eficácia em relação ao I em termos de menor preço ao consumidor final.

Como na maioria das indústrias de rede, a reestruturação das cadeias verticais nas indústrias de gás natural visa, em grande parte, facilitar a introdução de um maior grau de competição na cadeia produtiva. A consecução desse objetivo exige o exame do trade-off entre os potenciais benefícios esperados pelas pressões competitivas e os custos potenciais associados com os problemas contratuais que emergem, em muitos casos, da implementação de políticas visando à desintegração vertical das atividades econômicas.

Uma das ferramentas de políticas de introdução da concorrência em indústrias de rede é a reforma da estrutura de organização vertical das transações comerciais associada à separação contábil e/ou societária do atores atuantes em diferentes estágios da cadeia. $\mathrm{Na}$ indústria de gás natural, a introdução do segmento de carregadores independentes cumpriria a função de limitar o poder de mercado do segmento de transporte. Não obstante a presença de vários obstáculos à operação eficaz de um modelo de organização com a presença de carregadores, um resultado deste artigo aponta que a viabilidade deste modelo é função direta da estrutura de mercado no segmento de transporte.

E importante salientar que as decisões do regulador podem implicar, de fato, transferências de rendas entre diferentes agentes econômicos, sendo fácil de entender o porquê da ação de diferentes grupos de pressão que tentam influenciar o processo de tomada de decisões.

Dessa forma, duas questões de investigação merecem atenção particular neste trabalho: Qual a influência da entrada de comercializadores no processo de formação e apropriação das rendas? De que forma a organização vertical altera a repartição das rendas econômicas na cadeia de valor e o preço ao consumidor final? 
O presente artigo está organizado em quatro seções além desta introdução. Na segunda seção é apresentado o problema de organização vertical versus o duplo mark-up na perspectiva dos setores de infra-estrutura. A terceira apresenta os dois modelos básicos de organização vertical da indústria de gás natural através das variáveischave para a formação de preço dos agentes e de repartição da renda gerada na cadeia. Na quarta seção, são investigados os critérios para comparação e avaliação de desempenho dos dois modelos, em termos do preço ao consumidor e da repartição das rendas. Por fim, a quinta seção faz um balanço das principais conclusões deste artigo e como tais argumentos fornecem subsídios à análise de política setorial.

\section{Organização vertical de cadeias produtivas e o efeito do duplo mark-up}

O problema da apropriação das rendas econômicas ao longo de cadeias produtivas tem suscitado um forte interesse de investigação, em particular após os processos de reforma das indústrias de rede. $\mathrm{Na}$ presença de uma empresa verticalizada e monopolista, não restam dúvidas de que uma parcela substancial da renda de monopólio é apropriada pela firma, cabendo ao regulador fixar tarifas que reflitam a repartição destas rendas entre a firma e os consumidores.

A situação se torna mais complexa com a separação das atividades econômicas, como resultado de políticas de unbundling, pois o montante da renda econômica gerada, ao longo da cadeia, é alterado de acordo com o tipo de estrutura de mercado vigente. As decisões que concernem às regras tarifárias e às regras de acesso tornam-se cruciais nestas situaçōes, pois significam, de fato, uma arbitragem no processo de repartição das rendas entre os diferentes atores econômicos. Além disso, os custos de regulação também se tornam mais elevados, dada a necessidade ampliada de informações confiáveis sobre a estrutura de custos dos agentes.

Cabe salientar que tanto em situações de integração vertical como em estruturas desverticalizadas, o regulador se depara com a necessidade de criar instrumentos que induzam as firmas a revelarem a sua estrutura de custos. Isto mostra que uma parcela das rendas econômicas de monopólio poderia ser assimilada como produto do controle das informações econômicas - especialmente em matéria de custos - pela empresa monopolista.

Vickers (1998) enfatiza este aspecto chamando atenção para o fato de que o problema de assimetria de informação do regulador 
gera possibilidades para a firma se apropriar das chamadas rendas de informação, as quais podem reduzir a eficiência econômica. Como as rendas de informação podem representar uma fronteira entre bem-estar privado e social, elas proporcionam uma razão fundamental para que as relações verticais adotadas pela firma com poder de mercado gerem perdas de eficiência alocativa. Neste sentido, cabe observar que a estrutura de propriedade da firma afeta as rendas informacionais e os ganhos de eficiência alocativa. Assim, Vickers (1998) destaca que a introdução de pressões competitivas pode ser uma forma de reduzir as rendas de informação.

$O$ fato de existirem mais empresas operando numa determinada cadeia produtiva não soluciona automaticamente os problemas de poder de mercado e de detenção à entrada. Aghion e Bolton (1992) abordam este ponto, examinando se os contratos ótimos entre compradores e vendedores detêm a entrada de novos players $\mathrm{e}$ se, do ponto de vista do bem-estar social, tais contratos poderiam ser considerados subótimos. A idéia básica é de que quando vendedor e-comprador assinam um contrato, eles passam a ter poder de mercado sobre o entrante.

Como salientam David e Percebois (2001), o problema da integração vertical/desintegração vertical das indústrias de rede é controverso na literatura. Duas correntes de análise podem ser identificadas. A primeira destaca que a importância da desintegração vertical combinada com a introdução da competição tem como efeito principal evitar o problema do hold-up e a redução do excedente dos consumidores. O argumento principal é que é mais fácil evitar o problema de subsídios cruzados se o transportador não está presente a jusante na cadeia, pois, neste caso, poderia haver maior transparência no processo de formação e de repartição das rendas.

A segunda corrente argumenta que a integração vertical é preferível, pois evita o problema conhecido como duplo mark up. Este último tem importantes conseqüências para os processos de restruturação das indústrias de rede, pois está estreitamente associado aos problemas conhecidos como práticas restritivas verticais. Por esta razão, optamos por uma breve digressão teórica para explicitar este ponto.

A presença de duas empresas monopolistas ao longo de uma cadeia produtiva gera a situação de duplo mark up, pois cada uma delas tenderá a fixar preços acima de seus respectivos custos marginais.

Suponha duas situações distintas. Na primeira, uma empresa de transporte e de distribuição, verticalmente integrada e monopolista, apresenta uma curva de demanda $D_{1}$ hipotética como na Figura la 
A empresa produz $Q^{*}$ ao custo marginal c e receita marginal RM. O lucro $\pi^{*}$ é igual ao produto do mark up unitário (preço menos custo marginal: $\mathrm{p}-\mathrm{c}$ ) de monopólio $\pi$ pela quantidade produzida $\mathrm{Q}^{*}$. A taxa de mark up que incide sobre os custos é definida como o percentual $\mathrm{k}$, sendo $\mathrm{p}=\mathrm{c}(1+\mathrm{k})$. A maximização do lucro do monopolista conduz, no exemplo abaixo, a um lucro igual a 16 e a uma quantidade produzida igual a 4, pois:

$$
\begin{aligned}
& P=D_{1}(Q)=10-Q \\
& d \pi / d Q=d(P Q-c Q) / d Q=0
\end{aligned}
$$

Substituindo P, pela expressão (1) e igualando a derivada de $\pi$ (Q) a zero, temos:

$Q^{*}=4, p^{*}=6$ e $\pi^{*}=16$. O lucro da firma monopolista é dado pela área sombreada da figura 1a. A taxa de mark up $\mathrm{k}$ neste caso é igual a $200 \%$.

Na segunda situação, a empresa de transporte (firma 2) comercializa o produto para uma empresa de distribuição (firma 1) também monopolista no seu mercado. A diferença com relação ao primeiro caso é que agora temos dois preços: $\mathrm{p}_{2}$, que é o preço cobrado pelo transportador e pago pelo distribuidor; $\mathrm{e}_{1}$, o preço faturado pelo distribuidor ao consumidor final. A firma transportadora não altera o seu mark up e, portanto, o preço $\mathrm{p}_{2}$ é idêntico ao exemplo anterior já inclui o mark up do transportador, sendo igual a $\mathrm{p}_{2}=\mathrm{c} .(1+\mathrm{k})$, ou seja, $\mathrm{p}_{2}=6$.

A curva de demanda para o consumidor final não é alterada. Por serem ambas monopolistas, elas praticam preços acima de seus custos marginais. Procedendo da mesma forma que no caso anterior para a maximização de lucros, os resultados encontrados são significativamente piores. A oferta $Q^{*}=Q_{1}=Q_{2}=2$ cai $50 \%$, os preços praticados no mercado final $\left(\mathrm{p}_{1}=8\right)$ crescem $33 \%$, e o somatório dos lucros das firmas monopolistas é $25 \%$ inferior do que no caso da firma integrada.

Neste caso, ante as mesmas curvas de demanda $\mathrm{D}_{1}$ e de Receita Marginal, o distribuidor monopolista adiciona um mark up no processo de formação dos preços; a empresa transportadora segue igualmente adicionando o mark up referente à sua atividade. Assim, tal situação caracteriza a presença de duplo mark up na cadeia produtiva e revela os fortes incentivos existentes para a verticalização das atividades. 


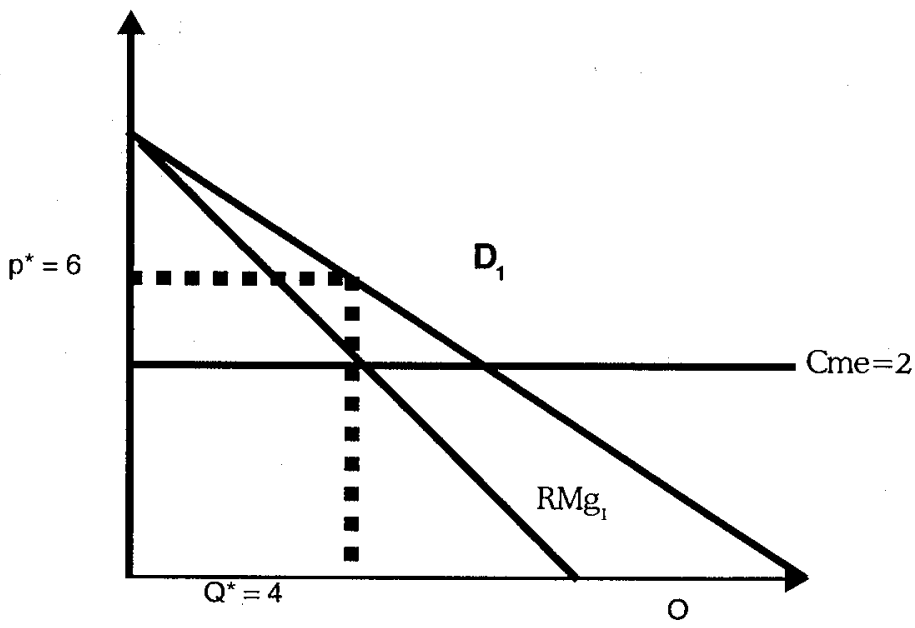

(a) Lucros de um produtor integrado

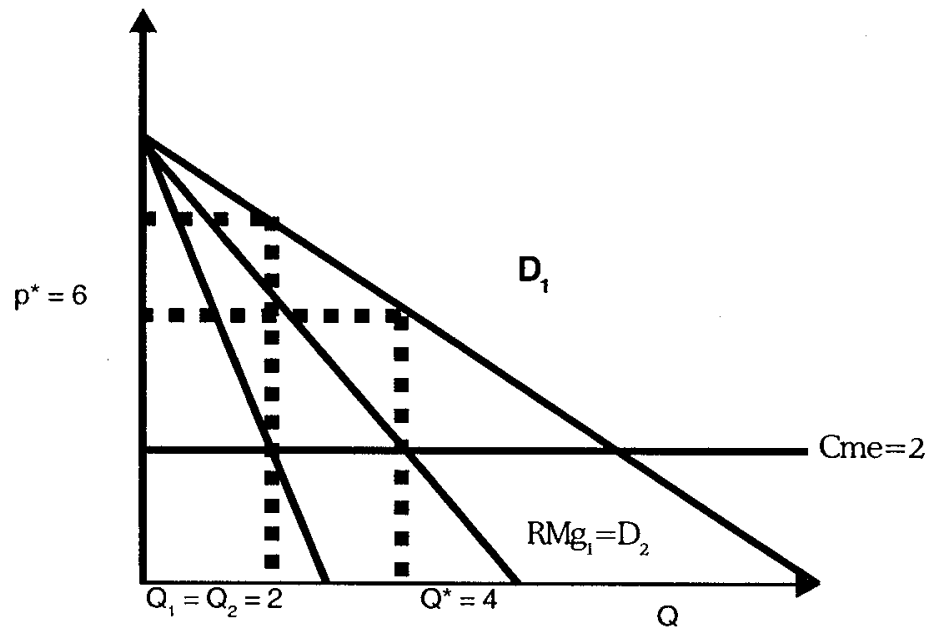

(b) Lucros de vários monopolistas

Figura 1: O caso do duplo mark up. 
Este problema apresentado acima constitui o elemento de decisão mais dificil para o processo de reformas estruturais nas indústrias de rede, pois não é nada trivial ponderar as vantagens e as desvan. tagens da integração vertical.

Cabe lembrar que, no caso da necessidade de expansão, importa igualmente considerar o grau de maturidade das redes e os aspectos geográficos que condicionam a configuração da rede. Estes aspectos são determinantes para a estrutura de contratos a ser estabelecidos entre os diferentes agentes econômicos. Curien (2000) destaca que é essencial entender as reformas recentes observadas em vários países como um conjunto de transformações dos modos de regulação e do sistema de concorrência nas indústrias de rede. Esse conjunto compreende:

- A liberalização/abertura à concorrência do mercado ou de alguns segmentos do mercado;

- A mudança do status do operador histórico da infra-estrutura de base (transmissão/transporte) através de privatização total ou parcial, ou da sua recomposição orgânica via desintegração vertical;

- A transformação da regulação, marcada pela separação funcional entre o exercício pretérito da tutela do Estado sobre a empresa, pelo controle do mercado e pela criação de um regime de incentivos.

Porém, o mesmo autor adverte que um fenômeno quase universal pode ser observado: o operador histórico, seja estatal ou privado, tenta sempre utilizar as condições de acesso às suas redes para manter (e eventualmente ampliar) a sua posição dominante. É precisamente por esta razão que, se o objetivo de política setorial é atrair um maior número de operadores, torna-se indispensável o distanciamento da empresa monopolista, proprietária dos ativos de transmissão/transporte, das atividades nos segmentos concorrenciais. Para atingir tal finalidade, o regulador pode impedir, inclusive, a participação cruzada de comercializadores, produtores e distribuidores no segmento de transporte. Este aspecto se constitui numa condição de neutralidade essencial para assegurar as condições eqüitativas de acesso às redes e tem sido adotado em países com redes maduras.

\section{Modelos de organização vertical e repartição da renda na indústria de gás natural}

A cadeia de produtiva do setor de gás natural pode ser abordada em três segmentos básicos: produção $(p)$, transporte $(t)$ e distribuiçāo $(d)$. No entanto, em alguns casos a organização vertical do fluxo financeiro das transações comerciais pode introduzir o seg- 
mento do carregador $(c)$, responsável pela intermediação entre produtores, transportadores e distribuidores.

Para análise do comportamento da relação entre mark-up sucessivos no setor de gás natural, definem-se dois modelos básicos gerados por duas formas organização vertical do fluxo comercial. A diferença marcante entre estes casos está na presença ou não do carregador independente. Quando não existe a figura do carregador, o agente do segmento de transporte é o agente intermediário não só o fluxo físico do produto entre produtores e distribuidores, mas também fica a cargo da intermediação financeira das transações comerciais.

É importante notar que a introdução do carregador quebra a linearidade do fluxo de comercialização; assim o transportador fica a cargo somente da intermediação física. Considere o modelo I como a representação dos casos que não existe a presença de carregadores, enquanto o modelo II simboliza a presença desse agente.

\section{Modelol}

A figura abaixo ilustra a organização do fluxo financeiro das transações comerciais no modelo de organização vertical I.

Produtor $\longrightarrow$ Transportador $\longrightarrow$ Distribuidor $\longrightarrow$ Consumidores

Será considerado que os agentes deste setor atuem em apenas um segmento da cadeia de valor, este é o caso mais geral e a situação de integração vertical entre setores é facilmente derivada deste.

Quanto à formação de preço dos agentes, são consideradas as seguintes variáveis:

Custo operacional unitário (Co $)$ - reflete o custo incorrido na operação de um determinado segmento da cadeia por unidade de produção. Assim, o produtor tem o determinado custo operacional unitário expresso por $\mathbf{C}^{\mathbf{o}} \mathbf{p}$, o transportador por $\mathbf{C}^{\circ} \mathbf{t}$ e o distribuidor por $\mathbf{C}^{\mathbf{0}} \mathbf{d}{ }^{1}$

Custo total unitário (C) - reflete a soma do custo operacional unitário ao custo de aquisição do gás natural do segmento a montante da cadeia. Neste caso, o produtor tem o custo operacional

1 No modelo II, com a presença do carregador, seu custo operacional será representado por $\mathrm{C}^{\circ} \mathrm{c}$. 
unitário igual ao custo total unitário. Já para o transportador o custo total unitário é igual $\mathbf{C}^{\mathbf{}}{ }_{\boldsymbol{t}}$ acrescido do preço pelo gás natural pago ao produtor $\left(\boldsymbol{P}_{p}\right)$. Analogamente, o distribuidor tem o custo total unitário é igual a $\boldsymbol{C}_{\boldsymbol{d}}=\boldsymbol{P}_{\boldsymbol{t}}+\boldsymbol{C}_{\boldsymbol{d}}^{\mathrm{o}}$.

De forma geral podemos expressar o custo total unitário de cada agentes $\left(\boldsymbol{C}_{\mathbf{i}}\right)$ da seguinte forma: $\mathrm{Ci}=P_{i-1}+C_{i}^{o}$, onde $i \in\{p=1, t=2$, $d=3\}$

Mark-up $(K)$ - Os preços são definidos pela aplicação de uma taxa de mark-up sobre os custos totais unitários. O mark-up é um valor percentual e, portanto, acrescido de um para efeitos de cálculo; ademais, o valor de $K$ reflete a condição estrutural (concentração) de cada segmento da cadeia de valor. ${ }^{2}$ Por exemplo, a formação de preço do transportador $\left(\boldsymbol{P}_{\boldsymbol{t}}\right)$ é dada por: $\mathbf{P}_{\mathbf{t}}=\left\{\mathbf{C}_{\mathbf{t}}^{\mathbf{o}}+\mathbf{P}_{\mathbf{p}}\right\} \mathbf{x}$ $\left(1+K_{t}\right)$.

De forma geral: $P_{1}=\left\{C_{i}^{o}+P_{i-1}\right\}\left(1+K_{i}\right)$

Margem liquida unitária (Mi) - a margem unitária é definida pela diferença entre o preço e o custo total unitário.

$$
M_{i}=P_{i}-C_{i}
$$

Ao analisar o processo de formação dos preços por mark-up, pode-se estabelecer uma relação direta com a estrutura do mercado. Portanto, a relação entre margem unitária e o mark-up pode ser expressa para um grande conjunto de situações, nas quais quanto maior o mark-up sobre os custos, maior a concentração do mercado.

Esta relação é usualmente apresentada nos manuais de análise microeconômica pela expressão que representa a formação de preços no modelo de Cournot com diferente número de firmas:

$$
\mathrm{P} / \mathrm{Cmg}=1 /\left[1-\frac{\mathrm{Si}}{\mathrm{Ed}}\right]
$$

Dada a elasticidade da demanda Ed, a mesma firma fixará um mark-up tão grande quanto maior sua participação relativa no mercado (Si). Nas situaçōes limites:

- quando $\mathrm{Si}=1$, ou seja, monopólio, a relação entre preço e custo marginal será expressa por;

- quando Si é infinitesimal, o preço tende ao custo marginal, situação típica de um mercado competitivo.

2 Este ponto será desenvolvido abaixo. 
Tendo visto a relação entre o mark-up e a estrutura dos mercados, vale avaliar a relação entre margem unitária, diferença entre o preço e o custo unitário total, e o mark-up.

A renda unitária total gerada $(R)$ na cadeia de valor é determinada pela soma das margens líquidas de cada agente.

$$
R=\sum_{i} M l_{i}
$$

Donde a participação na renda de cada agente é dada por:

$$
\mathrm{SRi}=M l_{i} / R \text {. }
$$

Logicamente, assim como o mark-up de cada agente é um elemento fundamental para explicar sua margem líquida unitária, a combinação dos mark-ups praticados na cadeia é um fator básico para determinar a arrecadação fiscal e a renda unitária total. Desta forma, para cada configuração da cadeia produtiva haverá um formato de repartição da renda no setor.

No modelo I, a margem líquida de cada agente é definida por:

Produtor: $M_{p}=C_{p}^{o} K_{p}$

Transportador: $M_{t}=K_{t}\left(C_{p}^{o}+C^{o}\right)+K_{t} K_{p} C_{p}^{o}$

Distribuidor:

$M_{d}=\left[K_{d} \times\left(C_{d}^{o}+C_{t}^{o}+C_{p}^{o}\right)\right]+\left[K_{d} \times K_{t} \times\left(C_{t}^{o}+C_{p}^{o}\right)\right]+\left[K_{d} K_{p} C_{p}^{o}\right]+\left[K_{d} K_{t} K_{p} C_{p}^{o}\right]$

Modelo II

A figura abaixo ilustra a organização do fluxo financeiro das transações comerciais no modelo de organização vertical II.

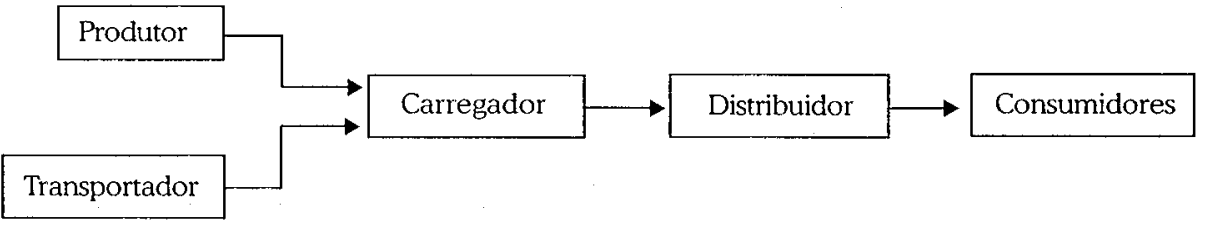


Neste caso, o fluxo de comercialização é diferente do fluxo físico do produto. Novamente será considerado que cada agente atue exclusivamente em determinado segmento.

Quanto à formação de preços, o método e as variáveis envolvidas são idênticos ao modelo I; porém, muda o encadeamento das transações. Com efeito, o transportador, deixa de ser um comprador do produtor e passa a ter seu custo total unitário unicamente determinado por seu custo operacional, a exemplo do produtor. Já o distribuidor deixa de ter como sua contraparte a montante o transportador e passa a negociar com o carregador. O carregador é a figura central nesta organização da cadeia, pois é o elo de ligação entre todos os segmentos.

Novamente, quanto mais a jusante na cadeia mais complexa é a relação entre a margem e o mark-up, sobretudo, esta relação incorporara as variáveis dos segmentos a montante. Porém, a diferença em relação ao modelo I é que, nesta configuração, a relação entre preço e mark-up para o transportador nāo incorpora as variáveis do produtor.

Produtor: $M_{p}=K_{p} C_{p}^{o}$

Transportador: $M_{t}=K_{t} C_{t}^{o}$

Carregador: $M_{c}=\left[K_{c}\left(C_{c}^{o}+C_{p}^{o}+C_{p}^{o}\right)\right]+\left[K_{c} K_{p} C_{p}^{o}\right]+\left[K_{c} K_{t} C^{o}\right]$

Distribuidor:

$M_{d}=\left[K_{d}\left(C_{d}^{o}+C_{c}^{o}+C^{o}{ }_{t}+C_{p}^{o}\right)\right]+\left[C_{p}^{o}\left(K_{d} K_{p}+K_{d} K_{c}+K_{d} K_{c} K_{p}\right)\right]+\left[C^{o}{ }_{t}\left(K_{d} K_{c}+K_{d}\right.\right.$ $\left.\left.K_{c} K_{t}\right)\right]+\left[K_{d} K_{c} C_{c}\right]$

\section{Critérios para avaliação de modelos de organização vertical da cadeia de valor}

Esta seção visa examinar o processo de formação da renda na indústria de gás natural. A partir dos modelos de organização da indústria, buscamos mensurar a renda econômica ao longo da cadeia e sua repartição entre os diferentes agentes econômicos. Duas condições distintas são investigadas neste procedimento: i) a repartição equânime ao longo da cadeia e ii) a condição de equivalência do preço final. Estas duas condições são apresentadas abaixo; em 
seguida, analisamos comparativamente os resultados obtidos com a utilização de cada um dos critérios.

\subsection{A condição de repartição equânime da renda ao longo da cadeia}

O equilíbrio na apropriação de rendas ao longo de uma cadeia de valor é um critério de análise relevante em qualquer atividade econômica, porém este aspecto é ainda mais crucial quando são estudados os setores de infra-estrutura, pois com presença de economias de rede, apresentam grande interdependência operacional e de investimentos entre seus agentes. No centro deste fenômeno está a presença de ativos essenciais (essential facilities) que não podem ser duplicados facilmente sem comprometer a viabilidade econômica das firmas.

$\mathrm{O}$ risco é que as estruturas verticalizadas propiciem a concentração (horizontal) de mercado, logo implicando a perda de eficiência alocativa. A complexidade de qualquer reforma institucional em indústrias de rede é como preservar os beneficios (externalidades positivas) das estruturas verticalizadas com a introdução da concorrência.

Uma assimetria muito acentuada na repartição da renda pode ser um indicador de ineficiência na organização vertical do setor, senão, pode propiciar um processo de verticalização da cadeia produtiva. Logicamente, existem diferenças na repartição da renda associada à natureza específica das operações de cada segmento na cadeia de valor. No entanto, para critérios de análise da organização vertical de um setor de infra-estrutura, a condição de repartição equânime das rendas configura-se em um importante parâmetro.

Isto posto, o que se busca discutir nesta seção é qual a condição para que a renda seja repartida igualmente entre os diferentes segmentos da cadeia de valor, ou seja, quando $S R_{i}=S R_{j}$ Neste intuito, apresenta-se a condição para $M l_{i}=M l_{j}$ em termos dos mark-up praticados pelos segmentos da cadeia de valor.

Avaliação da repartição equânime da renda na cadeia de valor da indústria de gás natural considera as seguintes hipóteses: i) o custo operacional do segmento de carregadores é zero, ou seja, ; ii) os custos operacionais dos demais agentes são idênticos, ou seja, . De fato, no segmento carregador custo por unidade transacionada tende a zero, pois não realiza operações industriais. Além disso, é fato estilizado no setor de gás natural que os custos operacionais sejam eqüitativamente distribuídos ${ }^{3}$ entre os estágios da cadeia de valor

3 Cabe salientar que, evidentemente, os custos fixos são bastante distintos segundo as características técnico-econômicas de cada um dos segmentos de atividade na cadeia do gás. 
(PINTO Jr. e KRAUSE, 2000a e 2000b). Desta forma, pode-se rescrever as equações de margem líquidas e principalmente definir qual a combinação de mark-ups ao longo da cadeia define uma repartição da renda equânime.

Os mark-ups da cadeia são expressos em termos do mark-up do produtor; assim, para o Modelo I vem que:

$$
\left(K_{d} K_{c} K_{p}\right)=\left[K_{p}, \frac{K_{p}}{\left(K_{p}+2\right)}, \frac{K_{p}}{\left(2 K_{p}+3\right)}\right]
$$

Para o Modelo II, analogamente, os mark-ups são:

$$
\left(K_{d} K_{c} K_{p}\right)=\left[K_{p}, K_{p}, \frac{K_{p}}{\left(2 K_{p}+2\right)}, \frac{K_{p}}{\left(2 K_{p}^{2}+9 K_{p}+6 / 2 K_{p}+2\right)}\right]
$$

Em ambos os casos, quanto mais a jusante na cadeia, menor deve ser o mark-up praticado pelo segmento da cadeia de valor para que se garanta a repartição equânime das rendas. Logicamente, isto é uma conseqüência direta do fenômeno de duplo mark-up. No entanto, no modelo II permite-se que os segmentos de produção e transporte pratiquem o mesmo mark-up sem prejudicar a repartição equânime das rendas, algo que não ocorreria no modelo I.

Como a diferença entre os dois modelos centra-se na introdução dos segmento carregador o importante é frisar a condição de renda equânime por $\mathrm{Kc}$.

Este valor crítico voltará a ser discutido na seção 4.3.

$$
K_{p}^{* *}=\frac{K_{p}}{\left(2 K_{p}+2\right)}
$$

\subsection{A condição de equivalência do preço final entre os modelos de organização vertical}

A forma de organização vertical da indústria, além da concentração de mercado em cada segmento horizontal da cadeia, condiciona o preço final ao consumidor de gás natural. O propósito desta seção é discutir a diferença do preço final nos dois modelos de organização vertical da indústria apresentados acima. Não obstante, esta comparação será feita simulando diferentes estruturas de mercado (concentração) nos segmentos verticais. 
O preço ao consumidor final no modelo I $\left(P_{d}^{I}\right)$ é expresso da seguinte forma:

$$
P_{d}^{1}=C_{d}+P_{t}+K_{d} C_{d}+K_{d} P_{t}
$$

O preço ao consumidor final no Modelo II $\left(\mathrm{P}_{d}^{\mathrm{II}}\right)$ é expresso da seguinte forma:

$$
P_{d}^{I I}=C_{d}+P_{c}+K_{d} C_{d}+K_{d} P_{c}
$$

A questão é avaliar a possibilidade de o modelo de organização II viabilizar a redução do preço final comparado com o modelo I. No entanto, cabe primeiramente exibir alguns cenários de preço final ao consumidor, considerando diferentes estruturas de concentração horizontal dos segmentos horizontais da cadeia produtiva.

Conforme abordado acima, a introdução do carregador independente pode repercutir em um preço maior ao consumidor final, diante disto, um critério de grande relevância na análise de modelos de organização vertical do setor de gás natural é inferir sobre a condição de equivalência do preço do distribuidor entre diferentes alternativas.

A condição para que a introdução do segmento de carregador traga benefícios em termos de redução do preço final do gás natural é dada pela seguinte restrição:

$$
P_{d}^{I I} \leq P_{d}^{I}
$$

Trabalhando na situação limite, ou seja, quando os preços finais são idênticos entre os dois modelos de organização vertical da indústria, segue a seguinte relação:

$$
\mathrm{C}_{d}^{o}+P_{t}+K_{d} C_{d}^{o}+K_{d} P_{t}=C_{d}^{o}+P_{c}+K_{d} C_{d}^{o}+K_{d} P_{c}
$$

logo:

$$
\begin{aligned}
& P_{t}+K_{d} P_{t}=P_{c}+K_{d} P_{c} \\
& P_{t}\left(1+K_{d}\right)=P_{c}+\left(1+K_{d}\right)
\end{aligned}
$$

donde a condição de equivalência do preço final é: $P_{t}=P_{c}$ 
A partir da condição expressa, é possível avaliar a estrutura do segmento de carregadores, simbolizado por $K c$, que justifique a adoção do modelo II na indústria.

A relação $P_{t}=P_{c}$ equivale a seguinte expressão:

$\left(C^{o}{ }_{t}+P_{p}+C^{o}{ }_{t} K_{t}+P_{p} K_{t}\right)=\left(C^{o}{ }_{c}+P_{p}+C^{o}{ }_{t}+K_{t} C^{o}{ }_{t}+K_{c} C_{c}^{o}+K_{c} P_{p}+K_{c} C^{o}{ }_{t}+K_{c} K_{t} C^{o}\right)$

Dado $P_{p}=C^{o}{ }_{t} K_{p}+C_{p}^{o}$, então:

$\left(C_{p}^{o} K_{t}+C_{p}^{o} K_{p} K_{r}\right)=\left(C_{c}^{o}+C_{c}^{o} K_{c}+C_{p}^{o} K_{c}+C_{p}^{o} K_{c}+C_{p}^{o} K_{p} K_{c}+C^{o}{ }_{t} K_{c}+C^{o} K_{t} K_{c}\right)$

logo:

$\left[C_{p}^{o}\left(K_{t}+K_{p} K_{t}\right)\right]-C_{c}^{o}=K_{c}+\left(C_{c}+C_{p}+C_{p} K_{p}+C_{t}+C_{t} K_{t}\right)$

Explicitando $K_{c}$ :

$$
K_{c}=\left\{\left[C_{p}^{o}\left(K_{t}+K_{p}+K_{t}\right)\right]-C^{o}\right\} /\left(C^{o}{ }_{c}+C_{p}^{o}+C_{p}^{o} K_{p}+C^{o}+C_{t}^{o}{ }_{t} K_{p}\right)
$$

Vale lembrar que (1) é a situação limite para que o modelo II de organização vertical da indústria de gás natural não repercuta em preços mais elevados ao consumidor final. Desta forma, a condição para a superioridade do modelo II de organização vertical da indústria em relação ao modelo I atende pela seguinte desigualdade:

Condição de eficiência do modelo II em termos do preço ao consumidor:

$$
K_{c}^{*} \leq\left\{\left[C_{p}^{o}\left(K_{t}+K_{p}+K_{t}\right)\right]-C_{c}^{o}\right\} /\left(C_{c}^{o}+C_{p}^{o}+C_{p}^{o} K_{p}+C^{o}{ }_{t}+C^{o}{ }_{t} K_{p}\right)
$$

A partir da expressão acima, identifica-se o mark-up do carregador; logo, ele é uma proxy da estrutura de mercado deste segmento, que permite resultados mais eficientes para o modelo II em termos de preço na ponta final da cadeia de valor. Portanto, trata-se de 
uma condição normativa para a adoção do modelo de organização vertical da indústria de gás natural que assume a presença de carregadores independentes.

Desta forma, pode-se exibir qual o mark-up máximo do segmento carregador que permite um resultado equivalente ao modelo I em termos do preço ao consumidor final. Para $K_{i} \in\{0,1,1\}$, com $i \in$ $\{p, t\}$ pode-se exibir a série de valores máximos de mark-up do segmento carregador $\left(K_{c}^{*}\right)$.

$\begin{array}{lll}K_{p} & K_{t} & K_{c}^{*} \\ 0,10 & 0,10 & 0,04 \\ 0,20 & 0,20 & 0,13 \\ 0,30 & 0,30 & 0,20 \\ 0,40 & 0,40 & 0,27 \\ 0,50 & 0,50 & 0,34 \\ 0,60 & 0,60 & 0,40 \\ 0,70 & 0,70 & 0,46 \\ 0,80 & 0,80 & 0,52 \\ 0,90 & 0,90 & 0,58 \\ 1,00 & 1,00 & 0,64\end{array}$

Quanto maior o mark-up dos segmentos de produção, transporte e distribuição, maior também será o mark-up máximo do carregador para que se obtenha um resultado equivalente no preço final do gás natural entre os modelos I e Il. Por outro lado, quando os mercados de cada segmento da cadeia (produção, transporte e distribuição) são pouco concentrados, exige-se um grande número de carregadores independentes para tonar eficiente a adoção do modelo II.

Além disso, o relevante é destacar a importância individual do segmento de transporte e de distribuição na determinação do markup máximo do segmento carregador capaz de gerar resultados eficientes. Desta forma, cabe apreciar as derivadas parciais de $K_{c}^{*}$ em relação a $K_{p}$ e a $K_{t}$.

$$
\begin{aligned}
& \partial K_{c}^{*} / \partial K_{p}=K_{t} \\
& \partial K_{c}^{*} / \partial K_{t}=1+K_{p} \\
& \text { se Ki } \in(0,1) \text { então } \\
& \partial K_{c}^{*} / \partial K_{t}=\partial K_{c}^{*} / \partial K_{p}
\end{aligned}
$$



o $K_{c}^{*}$

As tabelas abaixo ilustram a diferença entre $K_{p}$ e $K_{t}$ para explicar

\begin{tabular}{|c|c|c|c|c|c|c|c|}
\hline \multicolumn{4}{|c|}{ Relevância do segmento de transporte } & \multicolumn{4}{|c|}{ Relevância do segmento de transporte } \\
\hline$K_{p}$ & $\mathrm{~K}_{\mathrm{t}}$ & $\mathrm{K}_{\mathrm{c}}^{*}$ & $\operatorname{cresc} . \mathrm{K}_{\mathrm{c}}^{*}$ & $\mathrm{~K}_{\mathrm{p}}$ & $\mathrm{K}$ & $\mathrm{K}_{\mathrm{c}}^{*}$ & $\operatorname{cresc} \mathrm{K}_{\mathrm{c}}^{*}$ \\
\hline 0,10 & 0,10 & 0,04 & & 0,10 & 0,10 & 0,04 & \\
\hline 0,10 & 0,20 & 0,12 & 1,80 & 0,20 & 0,10 & 0,05 & 0,10 \\
\hline 0,10 & 0,30 & 0,19 & 1,55 & 0,30 & 0,10 & 0,05 & 0,08 \\
\hline 0,10 & 0,50 & 0,30 & 0,21 & 0,50 & 0,10 & 0,06 & 0,05 \\
\hline 0,10 & 0,50 & 0,30 & 0,21 & 0,50 & 0,10 & 0,06 & 0,05 \\
\hline 0,10 & 0,60 & 0,34 & 0,15 & 0,60 & 0,10 & 0,06 & 0,04 \\
\hline 0,10 & 0,70 & 0,39 & 0,12 & 0,70 & 0,10 & 0,06 & 0,04 \\
\hline 0,10 & 0,80 & 0,42 & 0,09 & 0,80 & 0,10 & 0,06 & 0,03 \\
\hline 0,10 & 0,90 & 0,45 & 0,08 & 0,90 & 0,10 & 0,07 & 0,03 \\
\hline 0,10 & 1,00 & 0,48 & 0,07 & 1,00 & 0,10 & 0,07 & 0,02 \\
\hline
\end{tabular}

Estes resultados ajudam a ilustrar a importância da estrutura do segmento de transporte em viabilizar o modelo II de organização vertical. Note que, mesmo com o crescimento do mark up dos produtores de $10 \%$ para $100 \%$, o mark-up máximo do segmento dos carregadores muda apenas de $4 \%$ para $7 \%$. Por outro lado, uma maior concentração do segmento de transporte justifica mais facilmente a introdução de modelo organizacional com a presença de carregadores independentes. Isto porque, para o mesmo intervalo de crescimento do mark-up dos produtores, no caso do transporte implica um mark-up máximo dos carregadores passando de $4 \%$ para $48 \%$. De fato, no primeiro caso, o crescimento de $K_{c}^{*}$ é de $55 \%$ enquanto, no segundo, chega a ser superior a $1000 \%$.

Outra forma de apresentar este resultado é em termos das retas de regressão linear entre $K_{c}^{*}$ e $K_{p}$ e entre $K_{c}^{*}$ e $K_{t}$, isto é, $K_{c}^{*}=\alpha K_{i}=\beta$. Donde:

Para $K_{i}=K_{p}$, temos $\alpha=0,0258$ e $\beta=0,0429$

Para $K_{i}=K_{t}$, temos $\alpha=0,4805$ e $\beta=0,0346$ 
A inclinação da curva que descreve o crescimento de $K_{c}^{*}$ é muito maior quando explicada por $K_{t}$ do que quando a variável índependente é $K_{p}$

Em suma, o que fica evidente é que o beneficio da introdução do modelo II de organização vertical da indústria está fortemente associado à relação entre a concentração vigente no segmento dos transportadores e à concentração do segmento dos carregadores. Mais precisamente, o mark-up máximo do segmento máximo aplicado pelos carregadores é uma fração do realizado pelos carregadores. Esta relação não é tão próxima na comparação com o segmento de produção. Desta forma, fica explícito que as condições normativas para a escolha de um modelo de organização vertical da indústria dependerão da atençã́o dos formuladores de política à estrutura de mercado do segmento de transporte e a restrição que este impõe ao segmento sobre a futura estrutura do segmento carregador para que a presença destes novos atores traga benefícios aos consumidores finais na cadeia de valor.

\subsection{Condição de equivalência do preço final versus condição de repartição equânime das rendas}

O intuito aqui é comparar o resultado da condição de eficiência da introdução do segmento carregador na cadeia de valor com a condição de repartição equânime das rendas. De fato, o interesse é apresentar o intervalo de valores do mark-up para o segmento carregador que atendam simultaneamente aos dois critérios.

Para tanto, o primeiro passo é rescrever a condição de equivalência do preço final. Considerando as hipóteses: i) o custo operacional do segmento de carregadores igual a zero, ou seja, $\mathrm{C}_{\mathrm{c}}^{\circ}=0$; ii); os custos operacionais dos demais agentes são idênticos, ou seja, $C_{p}^{o}=C_{t}^{o}=C_{d}^{o} \equiv C$. Isto posto, a condição de eficiência para adoção do modelo II pode ser expressa por:

$$
K_{c}^{*} \leq\left(K_{t}+K_{p} K_{t}\right) /\left(2+K_{p}+K_{l}\right)
$$

Como abordado acima, o mark-up do segmento carregador associado à repartição equânime das rendas é expresso por:

$$
K^{* *}=\frac{K_{p}}{\left(2 K_{p}+2\right)}
$$


Como na condição repartição equânime das rendas $K p=K t$, então pode-se reescrever este valor crítico por:

$$
K^{*}{ }_{c}=\frac{K_{t}}{\left(2 K_{t}+2\right)}
$$

As duas condições serão simultaneamente válidas quando o valor do mark-up do segmento carregador associado à repartição equânime da renda atenda a condição de eficiência do preço final, isto é, quando $\mathrm{K}^{* *}<\mathrm{K}_{\mathrm{c}}^{*}$.

Assim, a condição a ser verificada é:

$$
\frac{K_{t}}{\left(2 K_{t}+2\right)} \leq \frac{\left(K_{t}+K_{p} K_{t}\right)}{\left(2+K_{p}+K_{l}\right)}
$$

que equivale a seguinte expressão

$K_{t}^{2}+2 K^{2}{ }_{t} K_{p}+K_{t} K_{p} \geq 0$

que é verdadeira para todo $K_{t} \geq 0$ e $K_{p} \geq 0$

Logo, a condição de repartição equânime das rendas é adequada à condição de eficiência do modelo II. Este resultado é importante por exprimir que a organização vertical com a presença do segmento carregador, com repartiçăo da renda equânime, é mais eficiente do que o modelo I em termos de preços ao consumidor final. Portanto, ao perseguir o objetivo de política setorial da repartição da renda equânime, não só se está garantindo uma estabilidade e um equilíbrio de forças entre os agentes da cadeia, mas também igualmente a eficácia de modelos que contam com a presença do segmento carregador em promoverem menores preços ao consumidor final.

\section{Conclusão}

Alguns resultados podem ser derivados do tipo de análise desenvolvida neste artigo para se avaliar as políticas de concorrência no setor de gás natural, com foco nas mudanças na forma de organização vertical de sua cadeia de valor. O primeiro resultado, e o mais 
geral, é que a simples redefinição da forma de organização das relaçōes comerciais a partir de um modelo com carregadores independentes presentes não garante por si só a redução do preço ao consumidor final. De fato, o problema de mark-up duplo (sucessivos) pode repercutir maior preço ao consumidor final no modelo organizacional que conte com a presença de carregadores independentes (modelo II).

A eficácia deste modelo em promover a redução do preço, a jusante da cadeia, depende da estrutura de mercado dos segmentos de transporte e produção, com forte destaque para o primeiro. Considerando o mark-up aplicado pelos agentes como uma proxy do número de firmas atuando em cada segmento, para cada firma no segmento de transporte é necessário pelo menos 2 carregadores para que o resultado na ponta da cadeia seja eficiente em reduzir preços.

A metodologia de análise apresentada neste artigo fornece linhas gerais para análise do impacto de políticas de introdução da concorrência através de reforma na organização vertical da indústria.

Assim, as principais conclusões que podem ser tiradas a partir do artigo versam, em primeiro lugar, sobre a não existência de um modelo de organização vertical da indústria universalmente superior; ou seja, a desverticalização e a introdução do segmento de carregadores independentes não garantem por si maior eficiência alocativa.

De fato, a eficácia da introdução do carregador pelo modelo II depende fundamentalmente da estrutura de mercado do segmento de transporte. O mark-up praticado pelo segmento carregador deverá ser uma fração do exercido pelos transportadores em vias de gerar um resultado melhor em preços na ponta da cadeia em relação ao modelo I.

Por fim, foi discutido que a condição de repartição equânime das rendas é um critério relevante para análise de política setorial na medida em que fortes assimetrias entre os segmentos podem indicar a presença de ineficiência na organização vertical do setor.

\section{Referências bibliográficas}

AGHION, P., BOLTON, P., (1992), An Incomplete Contracts Approach to Financial Contracting, Review of Economic Studies, n.59, p. 473-94

CURIEN, N. (2000) LEconomie des Réseaux, La Découverte, collection Repères, 293, Paris.

DAVID, L. , PERCEBOIS, J., (2001) Les enjeux du transport pour le gaz et l'électricité: la fixation des charges d'accès, cahier n. 01,05.21, maio 2001, CREDEN LASER, Montpellier.

ELLIS, A. et. al. (2000) Structural Change in Europe's gas markets: three scenarios for the development of the European gas market to 2020. Energy Policy, n.28 p. 297-309 
ESTRADA, Javier, MOE Arild e MARTINSEN, Kare (1995), The Development of European Gas Markets: Environmental, Economic and Political Perspectives. John Wiley $\mathcal{G}$ Sons, Sussex, Inglaterra.

HELM, D. e JENKINSON, T. (1997) The Assessment: Competition in Regulated Industries Oxford Review of Econornic Policy, vol.13, n.1.

NEWBERY, D. M.G. (2000) Privatization, restructuring and regulation of network utilities. MIT Press, Cambridge(Mass.).

PINTO Jr. H.Q e KRAUSE, G.G.. (2000a): Estrutura e Regulação do Mercado de Gás Natural: experiência internacional. in Notas Técnicas - ANP, vol.I - Regulação. Nota Técnica n. 3.

PINTO Jr. H.Q e KRAUSE, G.G (2000b): Estrutura e Regulação do Mercado de Gás Natural: especificidades do caso brasileiro. in Notas Técnicas - ANP, vol.I - Regulação. Nota Técnican. 4.

SANTOS, R. T. (2001) Coordenação de Investimentos e Políticas de Introdução da concorrência na Indústria de Gás Natural: elementos para análise de casos no Brasil. Dissertação de Mestrado, Instituto de Economia - Universidade Federal do Rio de Janeiro.

VICKERS, J. (1998) Regulation, Competition and the Structure of Prices, in HELM, D., JENKINSON, T., Competition in Regulated Industries, Oxford University Press, 1998, p.2339.

WILLIAMSON, O. E. (1985) The Economic Institutions of Capitalism, New York: The Free Press.

WILLIAMSON, O.E (1997) Hierarchies, markets and power in the economy: na economic perspective in: MENARD, C. (1997) Transaction Cost Economics: recent developments. Edward Elgar Publishing Company 